\title{
The KEY to the end of the chemotherapy in advanced non-small cell lung cancer, or not yet?
}

\author{
Yiqing Huang ${ }^{1}$, Robert J. Walsh ${ }^{1}$, Ross A. Soo ${ }^{1,2}$ \\ ${ }^{1}$ Department of Haematology Oncology, National University Cancer Institute Singapore, Singapore, Singapore; ${ }^{2}$ Cancer Science of Institute, \\ National University of Singapore, Singapore, Singapore \\ Correspondence to: Ross A. Soo. Department of Haematology-Oncology, National University Cancer Institute Singapore, National University Health \\ System, 1E Kent Ridge Road, NUHS Tower Block Level 7, Singapore 119228, Singapore. Email: ross_soo@nuhs.edu.sg. \\ Comment on: Reck M, Rodríguez-Abreu D, Robinson AG, et al. Updated Analysis of KEYNOTE-024: Pembrolizumab Versus Platinum-Based \\ Chemotherapy for Advanced Non-Small-Cell Lung Cancer With PD-L1 Tumor Proportion Score of 50\% or Greater. J Clin Oncol 2019;37:537-46.
}

Submitted Apr 16, 2019. Accepted for publication Apr 28, 2019.

doi: $10.21037 /$ tlcr.2019.04.17

View this article at: http://dx.doi.org/10.21037/tlcr.2019.04.17

Immune checkpoint inhibitors (ICIs) have altered the treatment landscape of advanced non-small cell lung cancer (NSCLC) that lack driver mutations. In 2016, Reck and colleagues first reported the superiority of pembrolizumab versus chemotherapy in advanced NSCLC with PD-L1 tumour proportion score (TPS) $\geq 50 \%$ (KEYNOTE 024) (1). Patients with advanced NSCLC, high TPS of $\geq 50 \%$, and ECOG performance status of $0-1$ were eligible; while those with sensitizing EGFR mutations, $A L K$ rearrangements, or untreated brain metastases, were excluded. Progression free survival (PFS) was the primary end point, while overall survival (OS), objective response rates (ORR) and safety were key secondary end points. Crossover from chemotherapy to pembrolizumab arm was allowed at the time of progression. Based on the recommendation of the data and safety monitoring committee, the study was terminated early after second interim analysis showed an OS superiority with pembrolizumab. Pembrolizumab was associated with an improvement in PFS (10.3 vs. 6 months, $\mathrm{P}<0.001)$ and OS (estimated 6-month OS $80.2 \%$ vs. $72.4 \%, \mathrm{P}=0.005$ ) when compared with chemotherapy. This is despite a high initial crossover rate of $43 \%$ from chemotherapy arm to pembrolizumab. In addition, pembrolizumab was associated with a higher ORR (44.8\% vs. $27.8 \%)$ and less frequent grade 3 to 5 treatment related toxicities (27\% vs. 53\%) when compared with chemotherapy. Patients assigned to pembrolizumab also experienced improved quality of life and a delay to deterioration of symptoms (2).
In this update, the authors reported the updated OS and tolerability analysis. They included 3 statistical methods to adjust for potential bias introduced by crossover from chemotherapy to pembrolizumab. After a median follow-up of 25.2 months, survival doubled in the pembrolizumab arm compared to chemotherapy (30 vs. 14.9 months, nominal $\mathrm{P}=0.002$ ). At data cut-off, $54.3 \%$ of patients cross over from chemotherapy to receive pembrolizumab. Fifteen additional patients receive anti-PD1 treatment outside of crossover, making a crossover rate of $64.2 \%$ in the intention to treat (ITT) population (3).

Several findings are worth highlighting. Despite a high crossover rate, and analyses to adjust for potential bias with crossover, hazard ratios consistently favoured pembrolizumab arm. Overall survival benefit was maintained with the curves delineating clear separation on longer follow-up. In those who crossover from chemotherapy to receive $2^{\text {nd }}$ line pembrolizumab, the ORR was $20.9 \%$ : this result is similar to that of previous studies of $2^{\text {nd }}$ line antiPD1 treatment (4-6). Safety profile continued to favour pembrolizumab, with lower grade 3 to 5 treatment related adverse events ( $31 \%$ vs. $53 \%$ ) on longer follow-up.

Limitations to the current update include a relatively short median follow-up period of 25.2 months. This compares to a minimum 58.5 months follow-up reported in the updated CA209-003: a study of Nivolumab in pretreated NSCLC (7). In KEYNOTE 024, pembrolizumab could continue to 2 years and at the point of updated analysis, $11.0 \%$ of patients had completed therapy, while 
$19.9 \%$ remained on treatment. A longer follow-up will inform of outcomes after per protocol treatment cessation and provide robust long-term safety and efficacy data.

The three statistical models to adjust for effect of treatment crossover suffer inherent elements of error and accepted standard remains ITT analysis. Rank-preserving structural failure time (RPSFT) adjustment assumes common treatment effect of pembrolizumab regardless of when it is received, either first line or after crossover. While both inverse probability of censoring weighting (IPCW) and the simplified two-stage approach may be at increased risk of error due to the high crossover rate, and both assume the absence of unmeasured confounding factors. Despite these technical limitations, the three methods give similar adjusted HR for OS in the pembrolizumab arm $(0.49,0.52$, 0.52 for two-stage, TPSFT, IPCW respectively), suggesting a reliable result. Given the significant result in the ITT population, this adjustment analysis does not serve to alter the overall results of the study or treatment implications, but emphasises the significant benefit of pembrolizumab monotherapy in this population.

Several trials using single agent ICI in the treatmentnaive setting have been conducted (Table 1).

In CHECKMATE 026, a phase III study of nivolumab, no OS benefit was seen (HR 1.02) (10). Differences between KEYNOTE 024 and CHECKMATE 026 may be attributed to differences in patient population and PD-L1 assays (13). More recently, KEYNOTE 042 reported OS benefit with $1^{\text {st }}$ line pembrolizumab versus platinum-based chemotherapy in treatment-naïve NSCLC patients with PD-L1 TPS $\geq 1 \%$ (9). At a median follow-up of 12.8 months, OS benefit was seen across all subgroups: TPS $\geq 50 \%$ (20 vs. 12.2 months, $\mathrm{P}=0.0003$ ); $\geq 20 \%$ (17.7 vs. 13.0 months, $\mathrm{P}=0.002$ ); and $\geq 1 \%$ (16.7 vs. 12.1 months, $\mathrm{P}=0.0018$ ). Notably, patients with PD-L1 TPS $\geq 50 \%$ constituted approximately half of the entire cohort-a proportion much higher than the $30 \%$ seen in the general population (14). With the benefit largely driven by the high TPS group, this study highlights the benefit of single agent pembrolizumab in those with high TPS of $50 \%$. However, unlike KEYNOTE 024, patients with high PD-L1 TPS of $\geq 50 \%$ in KEYNOTE 042 did not show superiority in PFS for pembrolizumab compared to chemotherapy. Based on the results of KEYNOTE 042, the US FDA recently approved pembrolizumab for patients with advanced NSCLC expressing PD-L1 of at least $1 \%$.

Apart from single agent ICI, other studies evaluating ICIs with chemotherapy or with another ICI have been reported (Table 2).
In KEYNOTE 189, a phase III study of pembrolizumab and pemetrexed and a platinum compared with placebo and chemotherapy in non-squamous metastatic NSCLC, pembrolizumab and chemotherapy was associated with an improvement in OS (12-month OS $69.2 \%$ vs. 49.4\%, $\mathrm{P}<0.001)$ and PFS (8.8 vs. 4.9 months, $\mathrm{P}<0.001$ ), irrespective of $\mathrm{PD}-\mathrm{L} 1$ expression. Overall response rate in the pembrolizumab-combination and control arm was $47.6 \%$ and $18.9 \%$, respectively. In the subgroup of patients with TPS $\geq 50 \%$, a high ORR of $61 \%$ was seen in the pembrolizumab-combination arm (20). In a study of patients with advanced squamous NSCLC (KEYNOTE 407), pembrolizumab plus carboplatin and paclitaxel or nabpaclitaxel was associated with an improvement in OS (15.9 vs. 11.3 months, $\mathrm{P}<0.001)$ and $\mathrm{PFS}$ (6.4 vs. 4.8 months, $\mathrm{P}<0.001)$ compared to placebo plus chemotherapy (15). Once again, the benefit was seen across all PD-L1 categories. Both KEYNOTE 189 and KEYNOTE 407 have established the role of pembrolizumab and chemotherapy combination in $1^{\text {st }}$ line non-squamous and squamous NSCLC, respectively, regardless of PD-L1 expression.

Atezolizumab, an anti-PDL1 antibody, has also been studied with chemotherapy in advanced non-squamous and squamous NSCLC. In IMPOWER 130, the addition of atezolizumab to carboplatin plus nab-paclitaxel was associated with an improved PFS (7.0 vs. 5.5 months; $\mathrm{P}<0.0001)$ and $\mathrm{OS}$ (18.6 vs. 13.9 months, $\mathrm{P}=0.033$ ) compared with chemotherapy alone, with benefit seen across all PD-L1 subgroups (17). A study of atezolizumab with platinum and pemetrexed in non-squamous NSCLC also showed improved PFS (7.6 vs. 5.2 months, $\mathrm{P}<0.0001$ ) with addition of atezolizumab (18). At interim analysis, there was no difference in OS. In IMPOWER 150: a three-arm phase III study evaluating (I) atezolizumab and carboplatin and paclitaxel, (II) atezolizumab plus bevacizumab and carboplatin and paclitaxel, or (III) bevacizumab and carboplatin and paclitaxel in treatment-naive non-squamous advanced NSCLC (19), the study included a small proportion of patients with EGFR mutation and $A L K$ rearrangements. In the $W \mathrm{~T}$ population, an improvement in PFS (8.3 vs. 6.8 months, $\mathrm{P}<0.001)$ and OS (19.2 vs. 14.7 months, $\mathrm{P}=0.02)$ were seen with addition of atezolizumab to bevacizumab and chemotherapy compared with bevacizumab and chemotherapy. An improvement in PFS was also seen in the ITT population, including patients with EGFR mutations and ALK rearrangements, with a PFS of 8.3 vs. 6.8 months $(\mathrm{P}<0.0001)$. This study brings to surface the possible benefit of chemotherapyimmunotherapy combination in patients with oncogene 


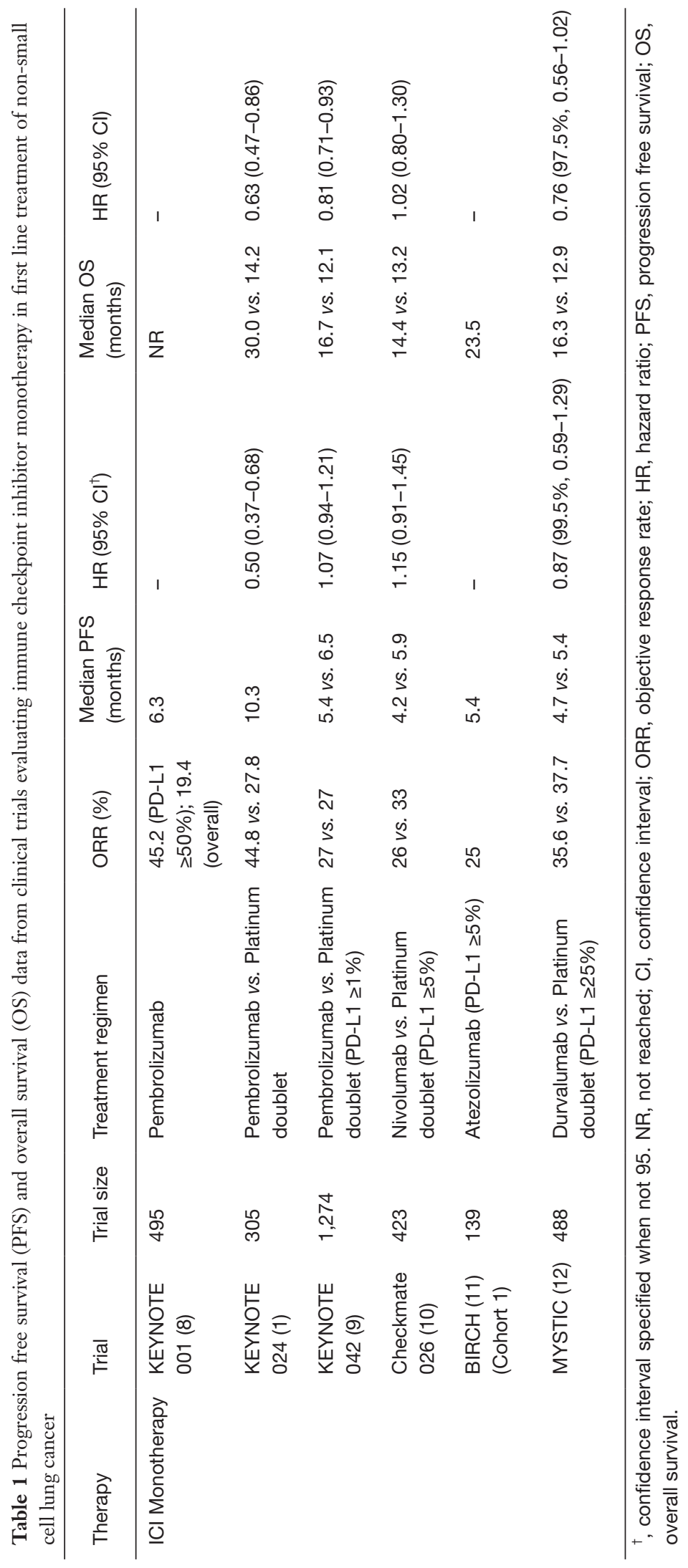




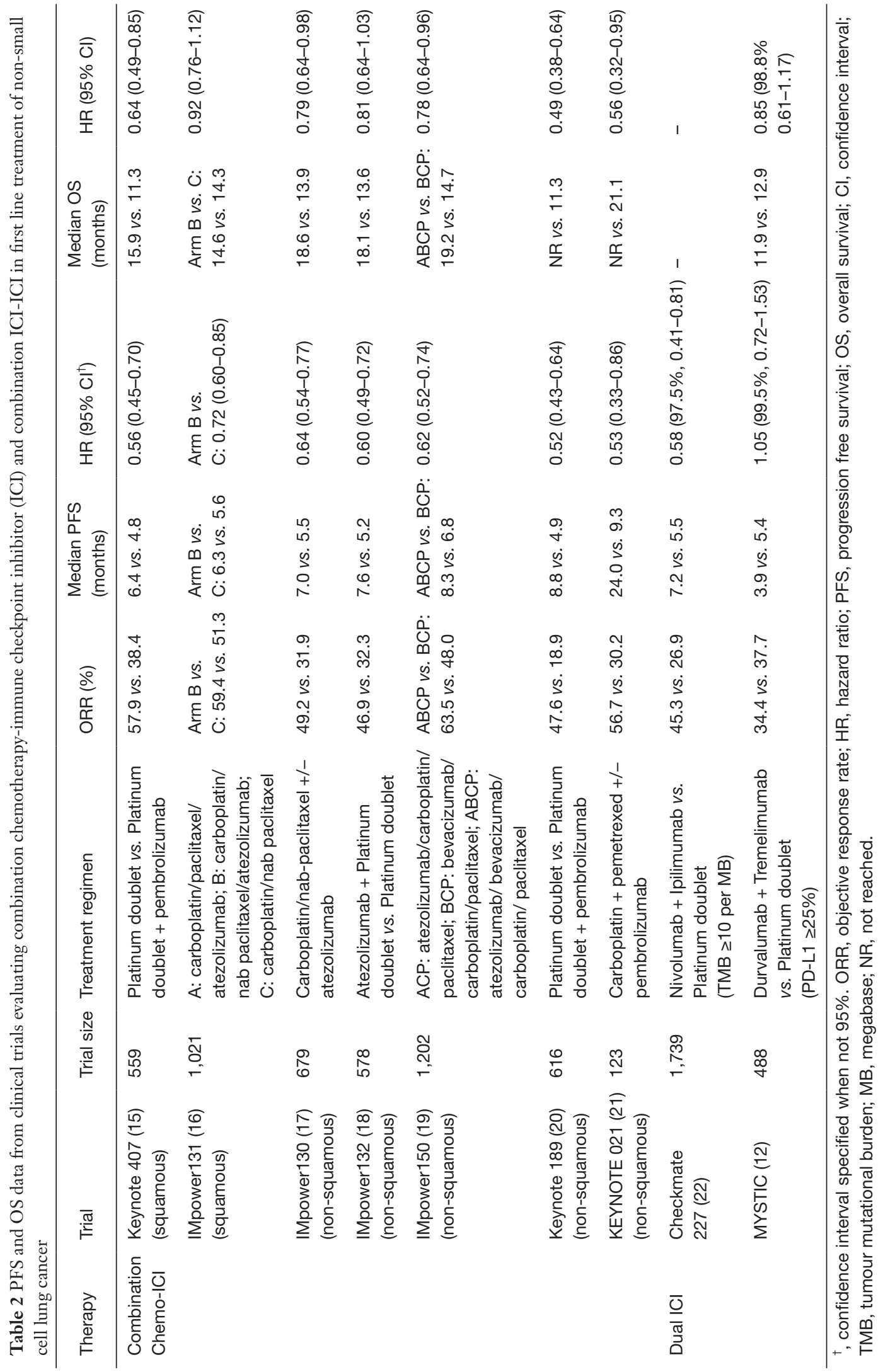


addicted NSCLC that have progressed on targeted therapies. Prospective large randomized controlled trials, however, are required to validate this. In IMPOWER 131 (advanced squamous NSCLC), there was an improvement in PFS for atezolizumab/carboplatin/nab-paclitaxel compared to chemotherapy (6.3 vs. 5.6 months, $\mathrm{P}=0.0001$ ) however, there was no difference in OS at interim analysis (23).

Dual ICIs in the $1^{\text {st }}$ line setting has also been reported. CHECKMATE 227 randomized patients with advanced NSCLC to platinum doublet chemotherapy, nivolumab plus ipilimumab, or either nivolumab monotherapy (in those PD-L1 $\geq 1 \%$ ), or nivolumab and chemotherapy (in those PD-L1 <1\%) (22). In patients with high tumour mutational burden (TMB), a PFS benefit was seen with nivolumab and ipilimumab, regardless of PD-L1 status (7.2 vs. 5.5 months, $\mathrm{P}<0.001)$. The ORR was also higher with combination immunotherapy in those with high TMB ( $45.3 \%$ vs. $26.9 \%$ ). Notably, at 1 year, patients treated with nivolumab and ipilimumab versus chemotherapy exhibited ongoing responses (68\% vs. 25\%). Nonetheless, longer follow-up and OS data are required. Preliminary results from CHECKMATE 227 of nivolumab with chemotherapy versus chemotherapy in those with PD-L1 $<1 \%$ have reported an improvement in PFS compared with chemotherapy alone (24).

Despite the positive data from KEYNOTE 024 update, several questions remain in clinical practice. With the establishment of pembrolizumab and chemotherapy combination as $1^{\text {st }}$ line treatment for NSCLC irrespective of PD-L1 expression $(15,20)$, should we be using pembrolizumab alone or chemo-immunotherapy combination for patients with high TPS $\geq 50 \%$ ? Is there still a subset of patients who will derive benefit from single agent pembrolizumab? Given the existing data, we believe single agent pembrolizumab should be considered in patients who are relatively asymptomatic. In patients who are symptomatic or have aggressive disease, a combination approach should be considered either pembrolizumab with chemotherapy, or a quadruplet regimen with atezolizumab, bevacizumab, carboplatin and paclitaxel, all of which have been approved by the US Food and Drug Administration in the $1^{\text {st }}$ line setting. Other combinations atezolizumab with a platinum plus a taxane or with pemetrexed but these combination have not been approved yet (18-20).

Whether pembrolizumab improves survival compared with chemotherapy in patients with PD-L1 TPS $<1-49 \%$ remains a question of clinical interest. In KEYNOTE
042, in an exploratory analysis of patients with PDL1 TPS $1-49 \%$, there was no difference in OS between pembrolizumab and chemotherapy (9). A phase II PEOPLE trial evaluating $1^{\text {st }}$ line pembrolizumab in advanced NSCLC with low PD-L1 ( $<50 \%)$ expression is currently ongoing (ClinicalTrial.gov identifier NCT03447678).

Several trials are examining combination ICIs and combinations of ICI and next generation immunotherapy. Such agents include vaccine based therapies (TG4010), LAG3 fusion protein, and tumour infiltrating lymphocytes (TILs) (ClinicalTrial.gov identifiers NCT03353675, NCT03625323, NCT03215810). The role of target lesion radiation therapy as an immune primer in combination with ICI is also under investigation (ClinicalTrials.gov identifier NCT03168464). With the ongoing expansion of the role of ICI in NSCLC, patient selection is key. Currently, PD-L1 remains the only approved biomarker in widespread clinical use. TMB appears a promising biomarker for benefit from ICI combination but is not yet in mainstream use (24). Treatment duration also requires further consideration, with significant clinical and financial toxicities associated with indefinite ICI use. CHECKMATE 153 showed improved PFS with continuous nivolumab until progression versus discontinuation at 1 year, with long term OS data awaited (25). The recruiting DICIPLE trial compares 6 months of combination ICI with re-challenge at progression versus continuation of combination ICI to progression (ClinicalTrials.gov identifier NCT03469960).

In conclusion, KEYNOTE 024 has established the role of single agent pembrolizumab in advanced NSCLC with high PD-L1 TPS $\geq 50 \%$, with continual OS benefit and favourable toxicity profile at longer follow-up. Combination of pembrolizumab and chemotherapy has also been established, with the benefit seen across all PD-L1 expression levels. We await more data and longer follow-up on other chemotherapy-immunotherapy combinations, and dual immunotherapy combinations.

\section{Acknowledgments}

None.

\section{Footnote}

Conflicts of Interest: RA Soo has received honorarium from Astra-Zeneca, BMS, Boehringer Ingelheim, Celgene, Lilly, Merck, Novartis, Pfizer, Roche, Taiho, Takeda, and Yuhan 
and research funding from Astra-Zeneca and Boehringer Ingelheim. The other authors have no conflicts of interest to declare.

\section{References}

1. Reck M, Rodríguez-Abreu D, Robinson AG, et al. Pembrolizumab versus Chemotherapy for PD-L1Positive Non-Small-Cell Lung Cancer. N Engl J Med 2016;375:1823-33.

2. Brahmer JR, Rodríguez-Abreu D, Robinson AG, et al. Health-related quality-of-life results for pembrolizumab versus chemotherapy in advanced, PD-L1-positive NSCLC (KEYNOTE-024): a multicentre, international, randomised, open-label phase 3 trial. Lancet Oncol 2017;18:1600-9.

3. Reck M, Rodríguez-Abreu D, Robinson AG, et al. Updated Analysis of KEYNOTE-024: Pembrolizumab Versus Platinum-Based Chemotherapy for Advanced NonSmall-Cell Lung Cancer With PD-L1 Tumor Proportion Score of 50\% or Greater. J Clin Oncol 2019;37:537-46.

4. Herbst RS, Baas P, Kim DW, et al. Pembrolizumab versus docetaxel for previously treated, PD-L1-positive, advanced non-small-cell lung cancer (KEYNOTE-010): a randomised controlled trial. Lancet 2016;387:1540-50.

5. Brahmer J, Reckamp KL, Baas P, et al. Nivolumab versus Docetaxel in Advanced Squamous-Cell Non-Small-Cell Lung Cancer. N Engl J Med 2015;373:123-35.

6. Borghaei H, Paz-Ares L, Horn L, et al. Nivolumab versus Docetaxel in Advanced Nonsquamous Non-Small-Cell Lung Cancer. N Engl J Med 2015;373:1627-39.

7. Gettinger S, Horn L, Jackman D, et al. Five-Year FollowUp of Nivolumab in Previously Treated Advanced NonSmall-Cell Lung Cancer: Results From the CA209-003 Study. J Clin Oncol 2018;36:1675-84.

8. Garon EB, Rizvi NA, Hui R, et al. Pembrolizumab for the Treatment of Non-Small-Cell Lung Cancer. N Engl J Med 2015;372:2018-28.

9. Mok TS, Wu YL, Kudaba I, et al. Pembrolizumab versus chemotherapy for previously untreated, PD-L1-expressing, locally advanced or metastatic non-small-cell lung cancer (KEYNOTE-042): a randomised, open-label, controlled, phase 3 trial. Lancet 2019. [Epub ahead of print].

10. Carbone DP, Reck M, Paz-Ares L, et al. First-Line Nivolumab in Stage IV or Recurrent Non-Small-Cell Lung Cancer. N Engl J Med 2017;376:2415-26.

11. Peters S, Gettinger S, Johnson ML, et al. Phase II Trial of Atezolizumab As First-Line or Subsequent Therapy for Patients With Programmed Death-Ligand 1-Selected Advanced Non-Small-Cell Lung Cancer (BIRCH). J Clin Oncol 2017;35:2781-9.

12. Rizvi NA, Chul Cho B, Reinmuth N, et al. LBA6Durvalumab with or without tremelimumab vs platinum-based chemotherapy as first-line treatment for metastatic non-small cell lung cancer: MYSTIC. Ann Oncol 2018. doi: http://dx.doi.org/10.1093/annonc/ mdy511.005.

13. Huang Y, Soo RA. The KEY to the end of chemotherapy in non-small cell lung cancer? Ann Transl Med 2017;5:166.

14. Smit EF, de Langen AJ. Pembrolizumab for all PD-L1positive NSCLC. Lancet 2019;393:1776-8.

15. Paz-Ares L, Luft A, Vicente D, et al. Pembrolizumab plus Chemotherapy for Squamous Non-Small-Cell Lung Cancer. N Engl J Med 2018;379:2040-51.

16. Jotte RM, Cappuzzo F, Vynnychenko I, et al. IMpower131: Primary PFS and safety analysis of a randomized phase III study of atezolizumab + carboplatin + paclitaxel or nab-paclitaxel vs carboplatin + nab-paclitaxel as $1 \mathrm{~L}$ therapy in advanced squamous NSCLC. J Clin Oncol 2018;36:LBA9000.

17. Cappuzzo F, Mekhail T, Zer A, et al. LBA53IMpower130: Progression-free survival (PFS) and safety analysis from a randomised phase III study of carboplatin + nab-paclitaxel $(\mathrm{CnP})$ with or without atezolizumab (atezo) as first-line (1L) therapy in advanced non-squamous NSCLC. Ann Oncol 2018;29.

18. Papadimitrakopoulou V, Cobo M, Bordoni R, et al. OA05.07 IMpower132: PFS and Safety Results with 1L Atezolizumab + Carboplatin/Cisplatin + Pemetrexed in Stage IV Non-Squamous NSCLC. J Thorac Oncol 2018;13:S332-3.

19. Socinski MA, Jotte RM, Cappuzzo F, et al. Atezolizumab for First-Line Treatment of Metastatic Nonsquamous NSCLC. N Engl J Med 2018;378:2288-301.

20. Gandhi L, Rodríguez-Abreu D, Gadgeel S, et al. Pembrolizumab plus Chemotherapy in Metastatic NonSmall-Cell Lung Cancer. N Engl J Med 2018;378:2078-92.

21. Borghaei H, Langer CJ, Gadgeel S, et al. 24-Month Overall Survival from KEYNOTE-021 Cohort G: Pemetrexed and Carboplatin with or without Pembrolizumab as First-Line Therapy for Advanced Nonsquamous Non-Small Cell Lung Cancer. J Thorac Oncol 2019;14:124-9.

22. Hellmann MD, Ciuleanu TE, Pluzanski A, et al. Nivolumab plus Ipilimumab in Lung Cancer with a High Tumor Mutational Burden. N Engl J Med 
2018;378:2093-104.

23. Socinski MA, Koynov KD, Berard H, et al.

LBA65IMpower131: Progression-free survival (PFS) and overall survival (OS) analysis of a randomised phase III study of atezolizumab + carboplatin + paclitaxel or nabpaclitaxel vs carboplatin + nab-paclitaxel in 1L advanced squamous NSCLC. Ann Oncol 2018;29.

24. Borghaei H, Hellmann MD, Paz-Ares LG, et al. Nivolumab (Nivo) + platinum-doublet chemotherapy (Chemo) vs chemo as first-line (1L) treatment $(\mathrm{Tx})$ for

Cite this article as: Huang Y, Walsh RJ, Soo RA. The KEY to the end of the chemotherapy in advanced non-small cell lung cancer, or not yet? Transl Lung Cancer Res 2019;8(5):731-737. doi: $10.21037 /$ tlcr.2019.04.17 advanced non-small cell lung cancer (NSCLC) with $<1 \%$ tumor PD-L1 expression: Results from CheckMate 227. J Clin Oncol 2018;36:9001.

25. Spigel D. 1297 O - Randomized results of fixed-duration (1-yr) vs continuous nivolumab in patients (pts) with advanced non-small cell lung cancer (NSCLC). Available online: https://www.primeoncology.org/app/uploads/ prime_activities/42584/VPS_Madrid_1297O_Lung_ Spigel.pdf 